\title{
Synthesis and characterization of thermotropic liquid crystalline polyimides
}

\author{
SACHIN MANE, C R RAJAN, SURENDRA PONRATHNAM and NAYAKU CHAVAN* \\ Polymer Science and Engineering Division, National Chemical Laboratory, Pune 411 008, India
}

MS received 23 April 2015; accepted 24 June 2015

\begin{abstract}
Non-symmetrical and linear dyad-based mesogens were synthesised containing imine or ester bridging group. In the present work, due to the absence of branching in diamine-based mesogen, the structure has-rigidity inversely imine/ester bridging groups between two benzene rings imparts-flexible property to the mesogen and consequently rigid-flexible property has been balanced. The synthesised mesogens were characterized by different techniques including nuclear magnetic resonance and Fourier transform infrared spectroscopy. Liquid crystalline polymers (LCPs) were synthesised using pyromellitic dianhydride and 4-[(4-aminobenzylidene)amino]aniline or 4-aminophenyl-4-aminobenzoate. Subsequently, thermotropic liquid crystalline polymers (TLCPs) have also been evaluated to obtain optical microscopy textures at different temperatures which demonstrated interesting and notable changes. It is worth noting that marble-like textures were observed upto $200^{\circ} \mathrm{C}$.
\end{abstract}

Keywords. Diamine; liquid crystalline polymer; mesogen; optical microscopy textures; pyromellitic dianhydride.

\section{Introduction}

In 1888, Australian botanist named Friedrich Reinitzer discovered $^{1}$ new phase of the material called as liquid crystal. However, liquid crystal is the intermediate state of the material to the isotropic liquid and crystalline solid. In isotropic liquid state, there is neither positional nor orientational molecule sequence. ${ }^{2}$ In contrast, crystalline state suffers through positional as well as orientational sequence of molecules. Many organic solid compounds when melt, do not convert into liquid phase, indicating that more than one transition form exist during physical state of transition. This indicates one or more intermediate phases exhibiting the properties of both solid as well as liquid. In 1922, French scientist $G$ Friedel classified the liquid crystals into three main types of mesogens ${ }^{3}$ depending on the order of molecules in the material, e.g., nematic, smectic and cholesteric.

Generally, liquid crystalline polymers (LCPs) consist of two basic units, rigid and flexible units. Although rigid part attributed to molecule's alignment, however, flexible part attributed to fluidity of the liquid crystal. The rigid part is known as mesogen and plays pivotal role in LCP texture. As a consequence, optimum balance of these two compositions is an essential during the synthesis of liquid crystalline polymer. In most of the cases, benzene is the backbone of LCPs due to high rigid property of benzene. Thermotropic liquid crystalline polymers (TLCPs) are formed during heating and further on cooling. LCPs have the potential applications ${ }^{4}$ in different fields, mostly in liquid crystal display. Padmavathy

\footnotetext{
*Author for correspondence (nn.chavan@ncl.res.in)
}

et $a l^{5}$ synthesised isocyanate-based mesogen for liquid crystal polymer synthesis. In another study, Mulani et $a l^{6}$ synthesised and characterized the azoxy-based mesogenic diols. In 2011, Chavan et $a l^{7}$ synthesised the cholesteric thermotropic liquid crystalline polyesters based on isosorbide. TLCPs have the potential applications to fabricate protective coatings, adhesives, structural foams and high strength composites. $^{8}$ TLCPs are widely used for protective coating in aerospace application wherein the use of current material is difficult. ${ }^{9}$ High-molecular-weight polymers are difficult to process due to viscosity. TLCPs are also used to process high-molecular-weight polymer which is not possible using conventional methods. ${ }^{10}$

Main objective of the present study is to synthesize the polymer and its evaluation to observe thermotropic optical textures. In the present work, two different thermotropic polymers were synthesised. To the best of our knowledge, TLC polyimides using pyromellitic dianhydride and 4-[(4-aminobenzylidene)amino]aniline or 4-aminophenyl-4aminobenzoate have not been synthesised at lower temperature of $200^{\circ} \mathrm{C}$. This work provides the TLC polyimides synthesis, characterization and texture evaluation by optical microscopy.

\section{Experimental}

\subsection{Materials}

4-Nitrophenol, 4-nitroaniline, 4-nitrobenzoic acid and 4nitrobenzaldehyde were procured from Loba Chemie, India. Stannous chloride, hydrochloric acid, toluene and N-methyl2-pyrrolidone (NMP) were purchased from Merck. Sulphuric 
acid was procured from Thomas Baker. Pyromellitic dianhydride (PMDA) was procured from Sigma-Aldrich. All chemicals were used as received.

\subsection{Characterization}

The synthesised mesogenic diamine was characterized by ${ }^{1} \mathrm{H}$ nuclear magentic resonance $\left({ }^{1} \mathrm{H} \mathrm{NMR}\right)(200 \mathrm{MHz})$ and Fourier transform infrared (FT-IR) spectroscopy. However, FT-IR spectra were recorded by Perkin Elmer instrument (Model: Spectrum GX and serial number: 69229). Number of scans were 10 and 1 interval. Optical microscopy was used to observe the liquid crystalline polymer textures.

\subsection{Reaction scheme}

In the present work, two non-symmetrical, linear and dyadbased mesogen, 4-[(4-aminobenzylidene)amino]aniline and 4-aminophenyl-4-aminobenzoate, were synthesised for the applications in LCPs synthesis. However, LCP synthesised from pyromellitic dianhydride and 4-[(4-aminobenzylidene) amino] aniline is represented in scheme 1, whereas LCP synthesised from pyromellitic dianhydride and 4-aminophenyl-4aminobenzoate is depicted in scheme 2 .

2.3a Synthesis of N,1-bis(4-nitrophenyl)methanimine (3): A dry, $500 \mathrm{ml}$, three-necked flask was fitted with a reflux condenser, thermometer and magnetic stirrer. To this, mixture of $10 \mathrm{~g}(66 \mathrm{mmol})$ of 4-nitrobenzaldehyde, $100 \mathrm{ml}$ of toluene, and 5-6 drops of $\mathrm{H}_{2} \mathrm{SO}_{4}$ were added. ${ }^{11}$ The solution was stirred and refluxed for $15 \mathrm{~min}$. To this solution, $9.12 \mathrm{~g}$ (66 mmol) of 4-nitroaniline was added. Subsequently, the reaction mixture was stirred for $5 \mathrm{~h}$ and refluxed in a nitrogen atmosphere. The completion of reaction was confirmed by TLC. Reaction mixture was cooled and filtered through Buchner funnel. The residual product was washed using an additional $20 \mathrm{ml}$ of toluene and dried in oven at $70^{\circ} \mathrm{C}$ under reduced pressure (yield: $16.5 \mathrm{~g}, 92 \%$ ).

2.3b Synthesis of 4-[(4-aminobenzylidene)amino]aniline (4): A dry, $500 \mathrm{ml}$, three-necked round bottom flask was fitted with magnetic stirrer, thermometer and a reflux condenser. To this, mixture of $15.38 \mathrm{~g}(55.304 \mathrm{mmol})$ of

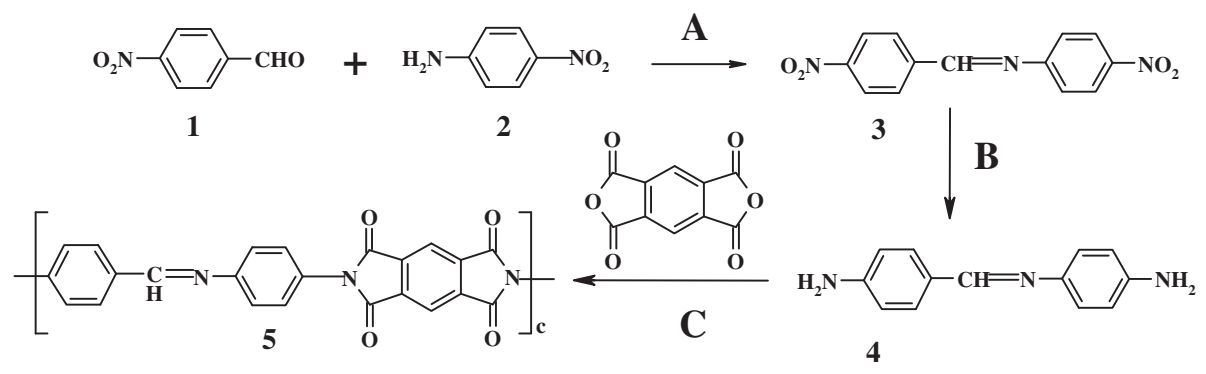

Scheme 1. Synthesis 4-[(4-aminobenzylidene)amino]aniline and liquid crystalline polyimide synthesis with pyromellitic dianhydride. 1: 4-nitrobenzaldehyde, 2: 4-nitroaniline, 3: N,1-bis(4-nitrophenyl)methanimine, 4: 4-[(4-aminobenzylidene)amino]aniline (ABAA), 5: PMDA + ABAA, A: $\mathrm{H}_{2} \mathrm{SO}_{4}$, toluene, $108^{\circ} \mathrm{C}, 5$ h, B: $\mathrm{FeSO}_{4} \cdot 7 \mathrm{H}_{2} \mathrm{O}, \mathrm{MeOH}+\mathrm{H}_{2} \mathrm{O}, \mathrm{KOH}$, reflux, $3 \mathrm{~h}, \mathbf{C :} 200^{\circ} \mathrm{C}, 15 \mathrm{~h}$.

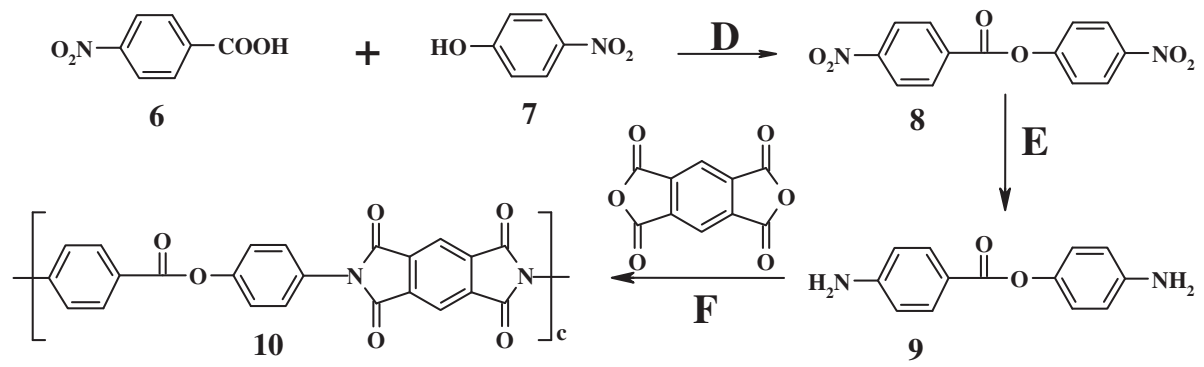

Scheme 2. Synthesis of 4-aminophenyl-4-aminobenzoate mesogen and liquid crystalline polyimide synthesis with pyromellitic dianhydride. 6: 4-nitrobenzoic acid, 7: 4-nitrophenol, 8: 4-nitrophenyl-4-nitrobenzoate, 9: 4-aminophenyl-4-aminobenzoate (APAB), 10: PMDA + APAB, D: $\mathrm{H}_{2} \mathrm{SO}_{4}$, IPA, 4 h, E: $\mathrm{FeSO}_{4} \cdot 7 \mathrm{H}_{2} \mathrm{O}, \mathrm{MeOH}+\mathrm{H}_{2} \mathrm{O}, \mathrm{KOH}$, reflux, 3 h, F: $200^{\circ} \mathrm{C}, 15 \mathrm{~h}$. 
$\mathrm{FeSO}_{4} \cdot 7 \mathrm{H}_{2} \mathrm{O}, 60 \mathrm{ml}(50 \%)$ of $\mathrm{MeOH}-\mathrm{H}_{2} \mathrm{O}(\mathrm{v} / \mathrm{v})$, and 5 $\mathrm{g}(18.4 \mathrm{mmol})$ of $\mathrm{N}, 1$-bis(4-nitrophenyl)methanimine were added. ${ }^{12}$ The mixture was stirred and refluxed for $30 \mathrm{~min}$. To this, $25 \mathrm{ml}$ of conc. $\mathrm{KOH}$ was added dropwise in 20 min and mixture was further refluxed for additional $20 \mathrm{~min}$ under stirring. The completion of reaction was confirmed by TLC. The mixture was transferred to a separatory funnel and the product was extracted using toluene $(2 \times 100) \mathrm{ml}$. The combined organic layer was washed with water, dried by magnesium sulphate, and toluene was evaporated. Slightly yellowish product was obtained. The product was dried in oven at $70^{\circ} \mathrm{C}$ under reduced pressure (yield: $3.427 \mathrm{~g}, 88 \%$ ).

2.3c Synthesis of 4-nitrophenyl-4-nitrobenzoate (8): A dry, $500 \mathrm{ml}$, three-necked flask was fitted with a reflux condenser, thermometer and magnetic stirrer. The flask was charged $^{13}$ with $10 \mathrm{~g}(59.84 \mathrm{mmol})$ of 4-nitrobenzoic acid, $100 \mathrm{ml}$ of isopropyl alcohol, and 2 drops of $\mathrm{H}_{2} \mathrm{SO}_{4}$ were added. This solution was stirred and refluxed for $20 \mathrm{~min}$. To this solution, $8.32 \mathrm{~g}$ (59.84 mmol) of 4-nitrophenol was added. The reaction mixture was stirred for additional $5 \mathrm{~h}$ under reflux in a nitrogen atmosphere. The completion of reaction was confirmed by TLC. The isopropyl alcohol was distilled out under vacuum. The reaction mixture was cooled and chloroform was added to residue and filtered through Buchner funnel under suction. The product was washed with an additional $10 \mathrm{ml}$ of chloroform and dried under reduced pressure at $70^{\circ} \mathrm{C}$ (yield: $15.35 \mathrm{~g}, 89 \%$ ).

2.3d Synthesis of 4-aminophenyl-4-aminobenzoate (9): A dry, $500 \mathrm{ml}$, three-necked round bottom flask was fitted with magnetic stirrer, thermometer and a reflux condenser. To this, mixture ${ }^{12}$ of $14.47 \mathrm{~g}(52.044 \mathrm{mmol})$ of $\mathrm{FeSO}_{4} \cdot 7 \mathrm{H}_{2} \mathrm{O}, 60 \mathrm{ml}$ $(50 \%)$ of $\mathrm{MeOH}-\mathrm{H}_{2} \mathrm{O}(\mathrm{v} / \mathrm{v})$ and $5 \mathrm{~g}(17.35 \mathrm{mmol})$ of $4-$ nitrophenyl 4-nitrobenzoate was added. The mixture was stirred and refluxed for $30 \mathrm{~min}$. To this, $25 \mathrm{ml}$ of conc. $\mathrm{KOH}$ was added dropwise in $20 \mathrm{~min}$ and refluxed for additional 30 min under stirring. The completion of reaction was confirmed by TLC. Furthermore, mixture was transferred to a separatory funnel to extract the product using toluene $(2 \times$ $100 \mathrm{ml}$ ). Finally, the organic layer was washed with water, dried by magnesium sulphate and toluene was evaporated. Slightly yellowish product was obtained. The product was dried at $70^{\circ} \mathrm{C}$ under reduced pressure (yield: $3.28 \mathrm{~g}, 83 \%$ ).

2.3e Synthesis of polyimides $(5,10): \quad \mathrm{LCP}^{14}$ of pyromellitic dianhydride with 4-[(4-aminobenzylidene)amino]aniline or 4-aminophenyl-4-aminobenzoate was synthesised. A dry, $50 \mathrm{ml}$, three-necked flask was equipped with a thermometer, nitrogen balloon, magnetic stirrer and dean stark apparatus. To this, $1.937 \mathrm{~g}(9.169 \mathrm{mmol})$ of 4-[(4aminobenzylidene)amino]aniline was dissolved in $5 \mathrm{ml}$ of N-methyl-2-pyrrolidone (NMP). Furthermore, 2 g (9.169 $\mathrm{mmol}$ ) of pyromellitic dianhydride was added and stirred for overnight to obtain viscous solution. Hereinafter $3 \mathrm{~h}$, reaction mixture was refluxed at $200^{\circ} \mathrm{C}$ for $15 \mathrm{~h}$. Water generated during polymerization process was allowed to remove through dean stark apparatus. Then, reaction mixture was cooled to ambient temperature. Subsequently, solution was diluted with NMP and slowly added to ethanol under stirring. The polymer was precipitated, filtered, washed with ethanol, and dried under reduced pressure at $100^{\circ} \mathrm{C}$. The yield of the polymer was $90 \%$.

Similar procedure was employed for the polymerization of 4-aminophenyl-4-aminobenzoate (2.093 g, $9.169 \mathrm{mmol})$ and pyromellitic dianhydride $(2 \mathrm{~g}, 9.169 \mathrm{mmol})$ with their respective composition. The yield of the polymer was $90 \%$. Synthesis of TLC polyimides is represented in schemes 1 and 2.

2.3f Preparation of TLC polyimides solution: LCP was processed to obtain the liquid crystalline textures. Herein, polyimide synthesised from pyromellitic dianhydride and 4-[(4-aminobenzylidene)amino]aniline was abbreviated as PMAB, whereas polyimide synthesised from pyromellitic dianhydride and 4-aminophenyl-4-aminobenzoate was abbreviated as PMAP. In this study, $40 \%$ solution of PMAB or PMAP was prepared in sulphuric acid for polymer dissolution to obtain TLCPs. Moreover, these polymers were observed under optical microscopy at different temperatures.

\section{Results and discussion}

In 1889, German physicist Otto Lehmann published ${ }^{15}$ the work related to the existence of liquid crystals. In the present work, two different diamine-based mesogens were synthesised and characterized by ${ }^{1} \mathrm{H}$ NMR and FT-IR spectroscopy.

\section{$3.1{ }^{1}$ H nuclear magnetic resonance (NMR)}

In this study, dyad-based achiral mesogen was synthesised for the application in LCP synthesis. Moreover, two dinitro compounds and further their reduced two diamino mesogens were characterized by ${ }^{1} \mathrm{H}$ NMR.

3.1a ${ }^{1} H$ NMR of N,1-bis(4-nitrophenyl)methanimine: A $N$,1-bis(4-nitrophenyl)methanimine was synthesised by condensation of 4-nitrobenzaldehyde and 4-nitroaniline and characterized by ${ }^{1} \mathrm{H}$ NMR. Results demonstrated that ${ }^{1} \mathrm{H}$ NMR (200 MHz, CD 3 OD, ppm): $\delta 6.59(4 \mathrm{H}, \mathrm{d})$ and $\delta 7.95$ (4H,d). ${ }^{1} \mathrm{H}$ NMR of $N, 1$-bis(4-nitrophenyl)methanimine is depicted in figure 1.

3.1b ${ }^{1}$ H NMR of 4-[(4-aminobenzylidene)amino]aniline: A 4-[(4-aminobenzylidene)amino]aniline was synthesised by reduction of $N, 1$-bis(4-nitrophenyl)methanimine and characterized by ${ }^{1} \mathrm{H}$ NMR. Results demonstrated that ${ }^{1} \mathrm{H}$ NMR $\left(200 \mathrm{MHz}, \mathrm{CDCl}_{3}+\mathrm{TMS}, \mathrm{ppm}\right) \delta 6.61(2 \mathrm{H}, \mathrm{d}), \delta 6.65(2 \mathrm{H}, \mathrm{d})$, 
$\delta 8.05(2 \mathrm{H}, \mathrm{d})$, and $\delta 8.10(2 \mathrm{H}, \mathrm{d}) .{ }^{1} \mathrm{H}$ NMR of $4-[(4-$ aminobenzylidene)amino]aniline is depicted in figure 2 .

3.1c ${ }^{1} H$ NMR of 4-nitrophenyl-4-nitrobenzoate: A 4nitrophenyl-4-nitrobenzoate was synthesised by condensation of 4-nitrobenzoic acid and 4-nitrophenol and characterized by ${ }^{1} \mathrm{H}$ NMR. Result demonstrated that ${ }^{1} \mathrm{H}$ NMR (200 $\left.\mathrm{MHz}, \mathrm{CD}_{3} \mathrm{OD}\right) \delta 8.2-8.6(8 \mathrm{H}, \mathrm{m}) .{ }^{1} \mathrm{H}$ NMR of 4-nitrophenyl4-nitrobenzoate is depicted in figure 3 .

3.1d ${ }^{1} H$ NMR of 4-aminophenyl-4-aminobenzoate: A 4aminophenyl-4-aminobenzoate was synthesised by reduction of 4-nitrophenyl-4-nitrobenzoate and characterized by
${ }^{1} \mathrm{H}$ NMR. Result demonstrated that ${ }^{1} \mathrm{H}$ NMR $(200 \mathrm{MHz}$, $\left.\mathrm{D}_{2} \mathrm{O}, \mathrm{ppm}\right): \delta$ 6.66-6.71 $(4 \mathrm{H}, \mathrm{d}), \delta$ 7.97-8.02 $(4 \mathrm{H}, \mathrm{d})$. However, peak of $-\mathrm{NH}_{2}(4.4-4.8)$ gets overlap with $\mathrm{D}_{2} \mathrm{O}$ peak (4.7-4.8). ${ }^{1} \mathrm{H}$ NMR of 4-aminophenyl-4-aminobenzoate is depicted in figure 4.

\subsection{FT-IR spectroscopy}

The mesogenic diamine was synthesised and also characterized by FT-IR (chloroform) spectrometer. Synthesis of $N, 1-$ bis(4-nitrophenyl)methanimine was confirmed ${ }^{16,17}$ by FT-IR $\left(\mathrm{CHCl}_{3}, \mathrm{~cm}^{-1}\right)$. FT-IR peak at 3019 corresponds to $\mathrm{C}-\mathrm{H}$ str. Furthermore, peaks at 756 and 929 were assigned to

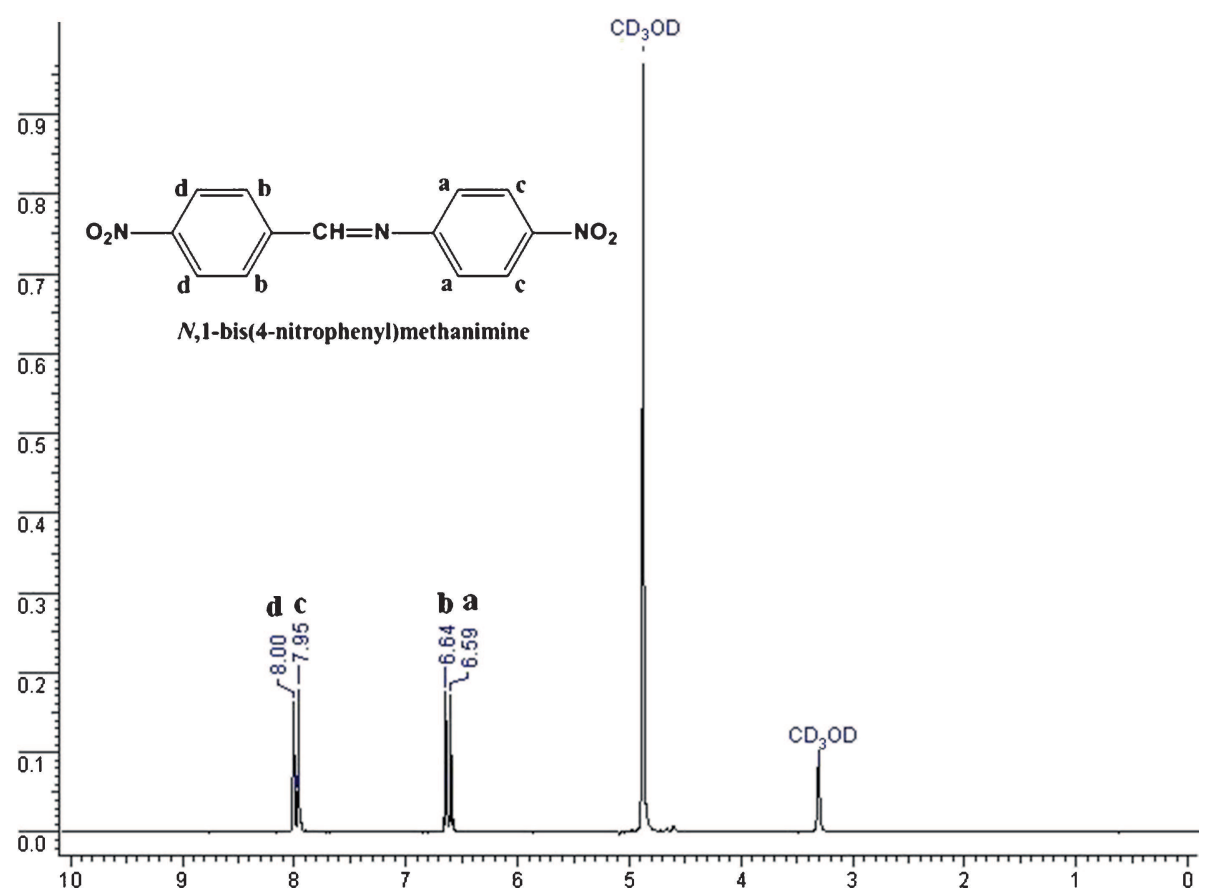

Figure 1. ${ }^{1} \mathrm{H} \mathrm{NMR}\left(200 \mathrm{MHz}, \mathrm{CD}_{3} \mathrm{OD}\right)$ of $N$,1-bis(4-nitrophenyl)methanimine.

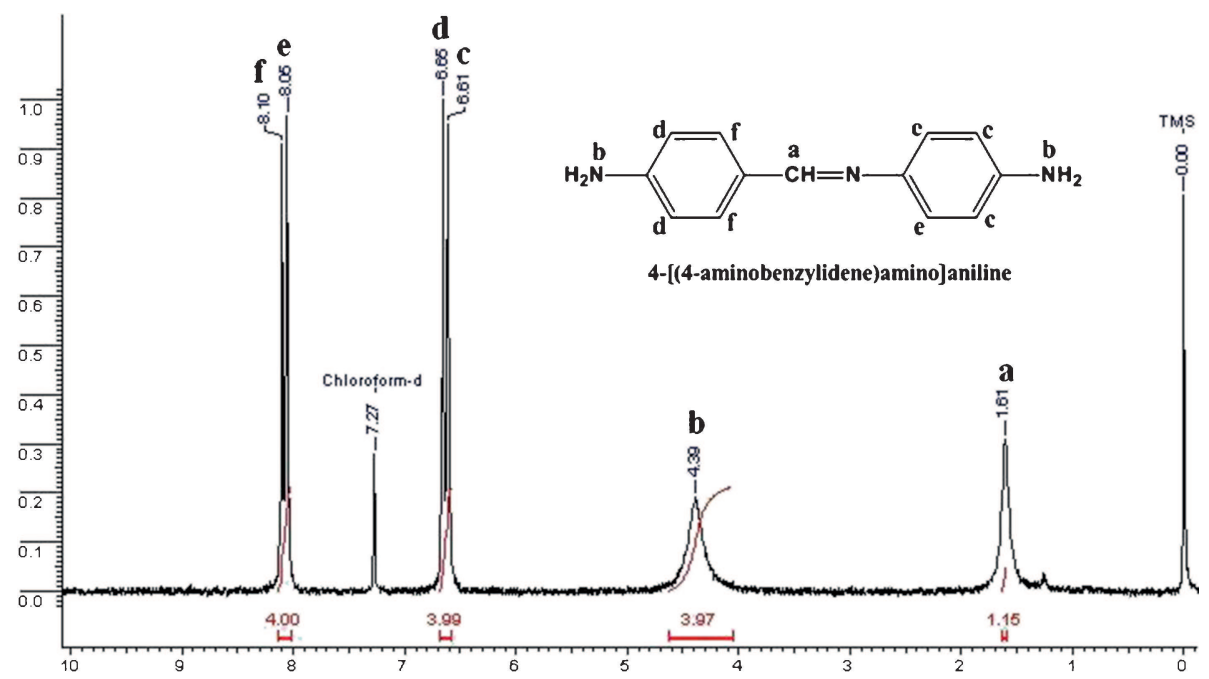

Figure 2. ${ }^{1} \mathrm{H} \mathrm{NMR}\left(200 \mathrm{MHz}, \mathrm{CDCl}_{3}+\mathrm{TMS}\right)$ of 4-[(4-aminobenzylidene)amino]aniline. 
aromatic $\mathrm{C}-\mathrm{H}$ out-of-plane bend. The peak observed at 1600 attributes to $\mathrm{C}=\mathrm{N}$ and 1519 peak assigned to $-\mathrm{NO}_{2}$ asymm. str. Hereinafter, peak at 832 assigned to paradisubstituted benzene ring. Furthermore, synthesis of 4-[(4aminobenzylidene)amino]aniline by nitro reduction of $N$, 1-bis(4-nitrophenyl)methanimine was also confirmed by FTIR $\left(\mathrm{CHCl}_{3}, \mathrm{~cm}^{-1}\right)$. Moreover, most confirmative peak of reduction was observed at 3418 assigned to $-\mathrm{NH}_{2}$ functionality. However, $-\mathrm{NO}_{2}$ asymm. str. peak at 1519 gets vanished which indicated the absence of nitro compound.
FT-IR spectra of $N$,1-bis(4-nitrophenyl)methanimine and $N$, 1-bis(4-nitrophenyl)methanimine are illustrated in figure 5. In another study, synthesis of 4-nitrophenyl 4-nitrobenzoate was confirmed ${ }^{18}$ by FT-IR $\left(\mathrm{CHCl}_{3}, \mathrm{~cm}^{-1}\right)$. The peak 3019 corresponds to $\mathrm{C}-\mathrm{H}$ str. vib., peak at 1629 assigned to ester functionality which is much lower due the presence of two phenyl ring either side of the ester. In addition, peak at 1530 attributes to $-\mathrm{NO}_{2}$ asymm. str. and peak 1349 assigned to $-\mathrm{NO}_{2}$ symm. str. Peak at 832 corresponds to para-disubstituted benzene ring. Peak observed at 929 and 755 assigned

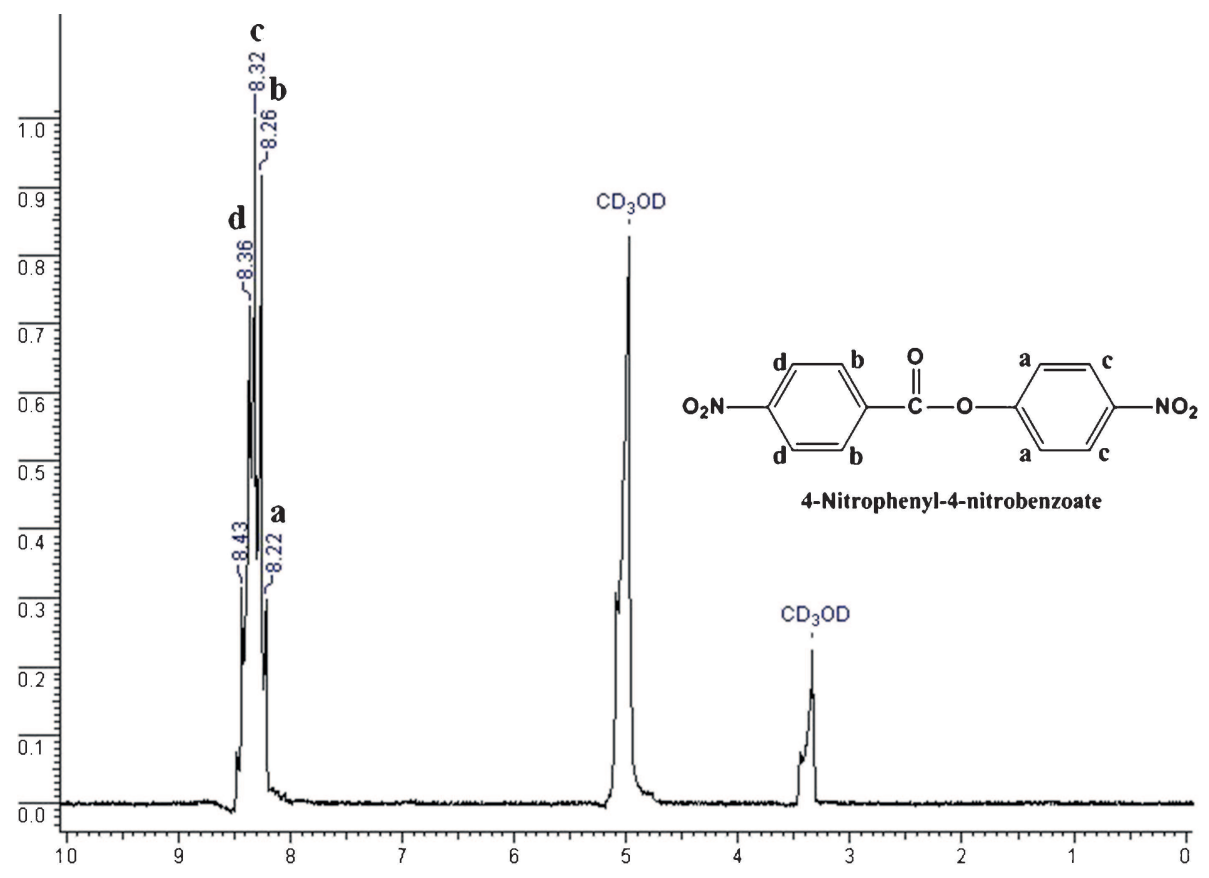

Figure 3. ${ }^{1} \mathrm{H} \mathrm{NMR}\left(200 \mathrm{MHz}, \mathrm{CD}_{3} \mathrm{OD}\right)$ of 4-nitrophenyl-4-nitrobenzoate.

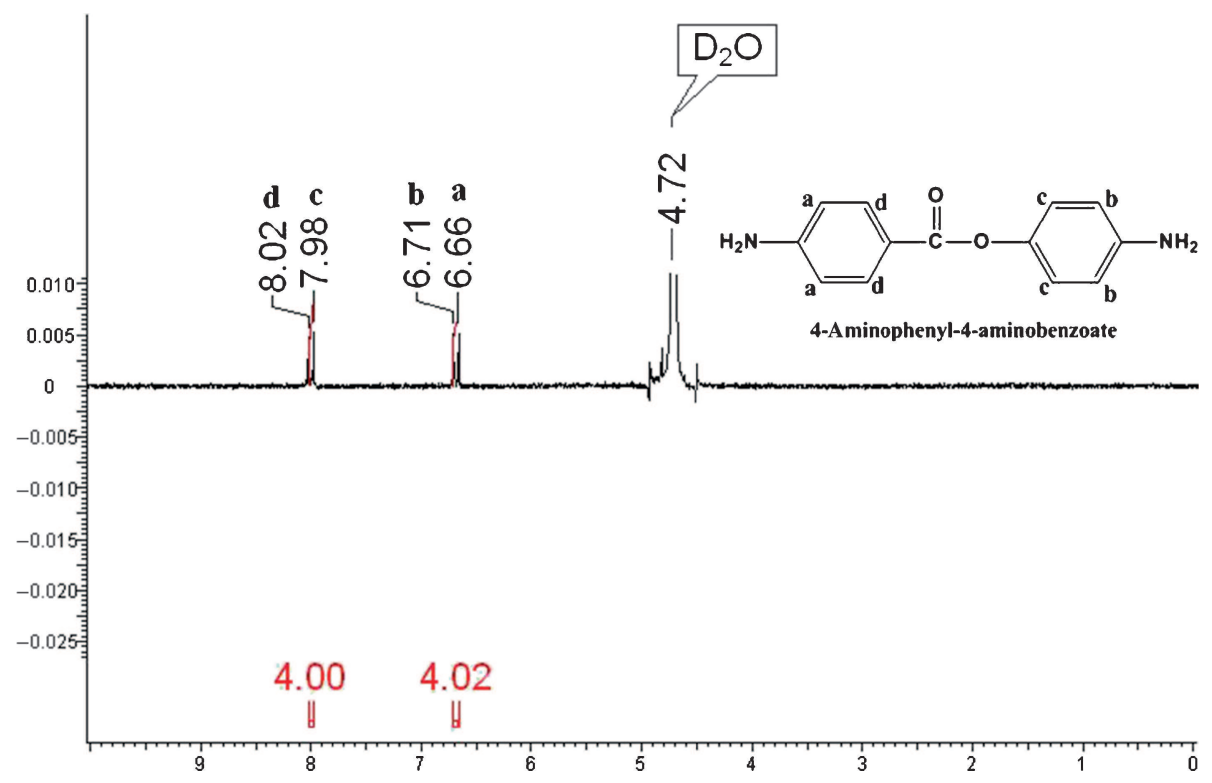

Figure 4. ${ }^{1} \mathrm{H} \mathrm{NMR}\left(200 \mathrm{MHz}, \mathrm{D}_{2} \mathrm{O}\right)$ of 4-aminophenyl-4-aminobenzoate. 


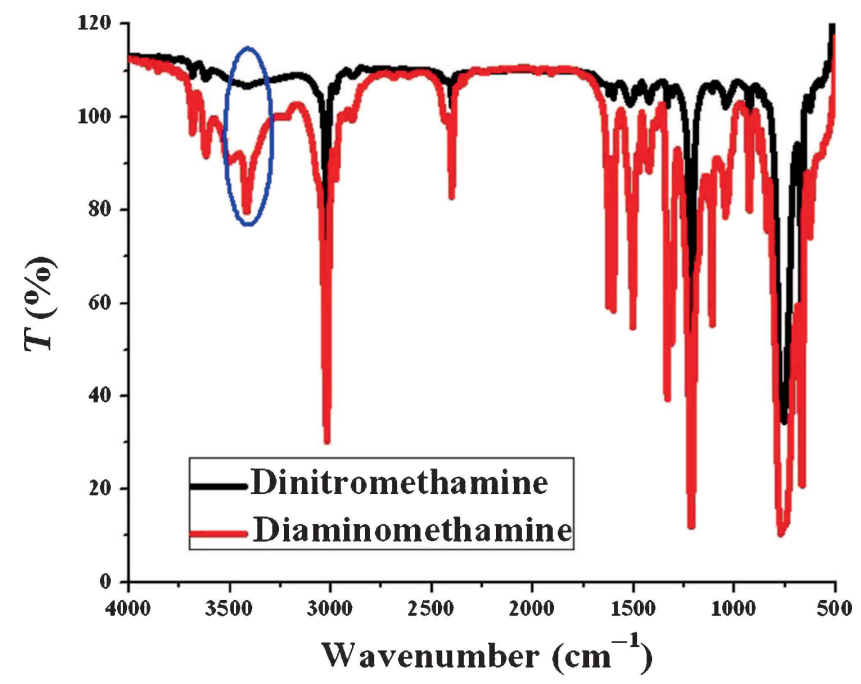

Figure 5. Comparative FT-IR spectrum of N,1-bis(4-nitrophenyl) methanimine and 4-[(4-aminobenzylidene)amino]aniline.

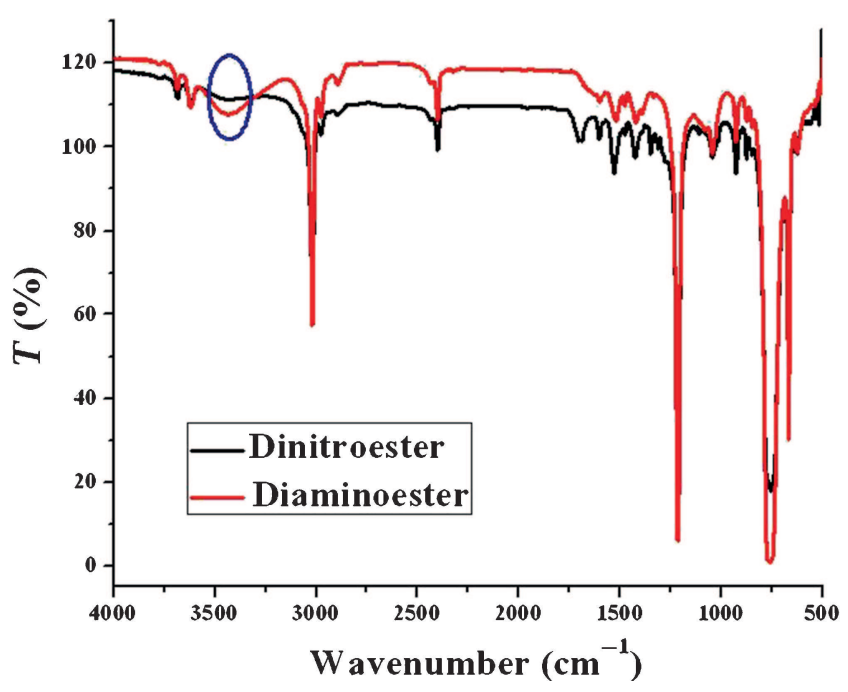

Figure 6. Comparative FT-IR spectrum of 4-nitrophenyl-4-nitrobenzoate and 4-aminophenyl-4-aminobenzoate.

to aromatic $\mathrm{C}-\mathrm{H}$ out of plane bend. Synthesis of 4-aminophenyl-4-aminobenzoate by reduction of 4-nitrophenyl-4nitrobenzoate was also confirmed by FT-IR $\left(\mathrm{CHCl}_{3}, \mathrm{~cm}^{-1}\right)$. Moreover, most confirmative peak of dinitro reduction was observed at 3435 corresponding to $-\mathrm{NH}_{2}$ functionality. In another, the absence of asymm. and symm. str. peak at 1530 and 1349 indicated the absence of $-\mathrm{NO}_{2}$ compounds. FT-IR spectra of 4-nitrophenyl-4-nitrobenzoate and 4-aminophenyl-4-aminobenzoate are represented in figure 6.

\subsection{Optical microscopy}

LCPs were synthesised using pyromellitic dianhydride and 4-[(4-aminobenzylidene)amino]aniline or 4-aminophenyl-4aminobenzoate. Furthermore, optical microscopy textures of LCPs were obtained at different temperatures with heating

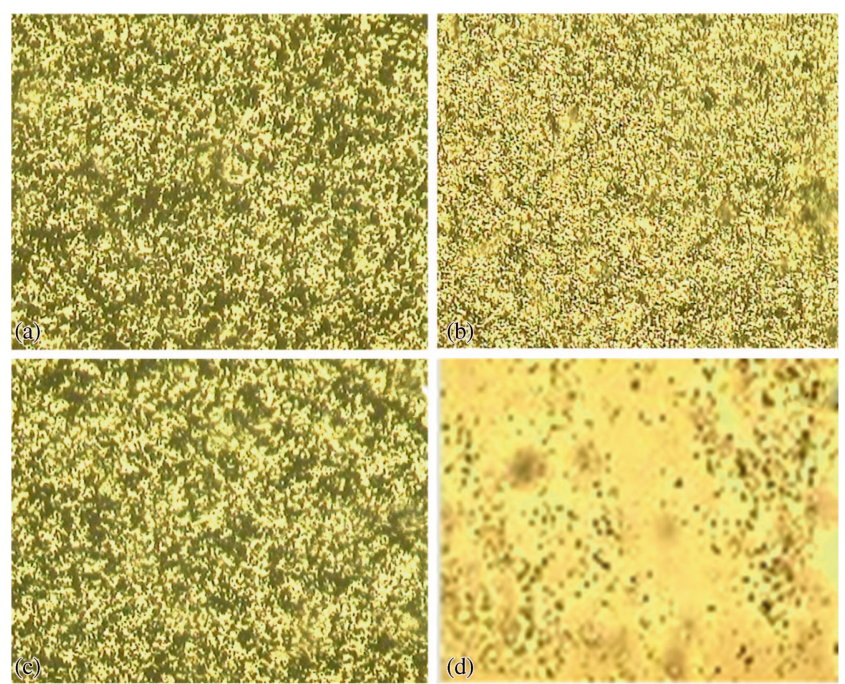

Figure 7. Polarized optical microscopic images of liquid crystalline polyimides of PMAB at (a) 181 , (b) $247^{\circ} \mathrm{C}$ and PMAP at (c) 187 and (d) $241^{\circ} \mathrm{C}$ temperatures.

rate of $10^{\circ} \mathrm{C} \mathrm{min}^{-1}$. For instance, changes of PMAB LCP (figure 7) was observed at (a) 181 and (b) $247^{\circ} \mathrm{C}$, whereas optical microscopy texture of PMAP was obtained at (c) 187 and (d) $241^{\circ} \mathrm{C}$. It is worth noting that, optical microscopy revealed the interesting textures after $200^{\circ} \mathrm{C}$. Notably, marble texture was observed upto $200^{\circ} \mathrm{C} .{ }^{19}$ These changes were observed at different temperature intervals. Optical microscopic textures at different temperatures are depicted in figure 7.

\section{Conclusions}

In conclusion, non-symmetrical and linear dyad-based mesogens were synthesised. Two different diamine-based mesogens, 4-[(4-aminobenzylidene)amino]aniline and 4-aminophenyl-4-aminobenzoate, were successfully synthesised and characterized by ${ }^{1} \mathrm{H}$ NMR and FT-IR spectroscopy. In addition to this, LCP of PMAB and PMAP was successfully synthesised at comparatively low temperature. Synthesised liquid crystal textures were observed by optical microscopy at different temperatures. Notably, marble texture background was observed upto $200^{\circ} \mathrm{C}$, whereas nematic-like texture was observed on further heating above $200^{\circ} \mathrm{C}$. These changes were observed at different temperatures, which revealed that temperature plays pivotal role in texture demonstration.

Abbreviations: PMDA: pyromellitic dianhydride; PMAB: pyromellitic dianhydride + 4-[(4-aminobenzylidene)amino] aniline; PMAP: pyromellitic dianhydride +4 -aminophenyl 4-aminobenzoate.

\section{Acknowledgements}

This research was financially supported by University Grant Commission (UGC), New Delhi, India. We gratefully 
acknowledge to the UGC for the award of research fellowship (Fellowship Award no.: Sr. No.: 2061010407; Ref. No. 20-06/2010(i)EU-IV).

\section{References}

1. Laschat S 2009 J. Org. Chem. 51

2. Stariolo D A and Barci D G 2010 J. Phys.: Conf. Ser. 246 012021

3. Vlad P N, Gerlic I and Kralj S 2009 Int. J. Mol. Sci. 103971

4. Liu D and Broer D J 2014 Langmuir 3013499

5. Padmavathy T, Srinivasan K S V and Venukateswarulu U 2001 Mol. Cryst. Liq: Cryst. 37835

6. Mulani K B, Ganjave N V and Chavan N N 2014 Indian J. Chem. 53B 359

7. Chavan N N 2011 Mater. Sci. Appl. 21520

8. Economy J and Goranov K 1994 High Perform. Polym. 117 221

9. Guerriero G, Alderliesten R, Dingemans T and Benedictus R 2011 Prog. Org. Coat. 70245
10. Tang Y and Gao P 2012 Viscoelasticity-from theory to biological applications, micro-rheological study on fully exfoliated organoclay modified thermotropic liquid crystalline polymer and its viscosity reduction effect on high molecular mass polyethylene (InTech) Chapter-13, p 213

11. Barot K P, Manna K S and Ghate M D 2013 J. Saudi Chem. Soc. doi:10.1016/j.jscs.2013.09.010

12. Aliyan H, Fazaeli R, Naghash H J, Massah A R and Saliminejad F 2012 J. Nanostruct. 121

13. Williams G and Clark D J 1956 J. Chem. Soc. 2651304

14. Ekincki E, Koytepe S, Pasahan A and Seckin T 2006 Turk. J. Chem. 30277

15. Lehmann O 1889 Z. Phys. Chem. 4462

16. Kalaichelvan S, Sundaraganesan N and Joshua B D 2008 Indian J. Pure Appl. Phys. 47A 1632

17. Kavitha E, Sunderganesan $\mathrm{N}$ and Sebastian S 2010 Indian J. Pure Appl. Phys. 4820

18. Samsonowicz M, Swislocka R, Regulska E and Lewandowski W 2007 Int. J. Quantum Chem. 107480

19. Park Y S, Lee J W and Jin J I 2002 Bull. Korean Chem. Soc. 231201 